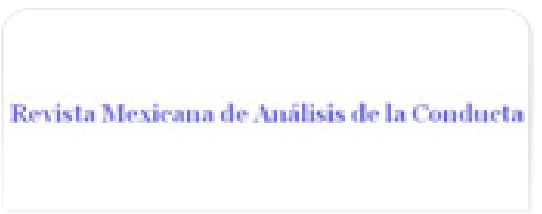

Revista Mexicana de Análisis de la Conducta ISSN: 0185-4534

editora@rmac-mx.org

Sociedad Mexicana de Análisis de la Conducta

México

JIMÉNEZ, ÁNGEL ANDRÉS; CABRERA, FELIPE; COVARRUBIAS, PABLO ELECCIÓN DE MODOS DE ACCIÓN Y MÉTRICA INTRÍNSECA EN UNA TAREA DE ALCANCE CON EL BRAZO

Revista Mexicana de Análisis de la Conducta, vol. 40, núm. 1, junio, 2014, pp. 1-24 Sociedad Mexicana de Análisis de la Conducta Distrito Federal, México

Disponible en: http://www.redalyc.org/articulo.oa?id=59335810001

Cómo citar el artículo

- Número completo

- Más información del artículo

- Página de la revista en redalyc.org

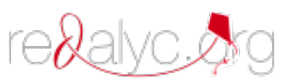

Sistema de Información Científica Red de Revistas Científicas de América Latina, el Caribe, España y Portugal Proyecto académico sin fines de lucro, desarrollado bajo la iniciativa de acceso abierto 


\title{
ELECCIÓN DE MODOS DE ACCIÓN Y MÉTRICA INTRÍNSECA EN UNA TAREA DE ALCANCE CON EL BRAZO
}

\author{
CHOICE OF MODES OF ACTION AND INTRINSIC METRIC IN AN \\ ARM REACHING TASK
}

\section{ÁNGEL ANDRÉS JIMÉNEZ, FELIPE CABRERA Y PABLO COVARRUBIAS UNIVERSIDAD DE GUADALAJARA, CUCI. CENTRO DE INVESTIGACIÓN EN CONDUCTA Y COGNICIÓN COMPARADA}

\begin{abstract}
Resumen
Dos grupos de participantes, hombres y mujeres, alcanzaron en posición sedente un bloque de plástico que se presentó sobre una mesa a diferentes distancias. Cuando la distancia se expresó en unidades extrínsecas (centímetros), las mujeres cambiaron sus modos de acción al alcanzar el bloque a distancias más cercanas que los hombres debido a que sus dimensiones corporales fueron menores que las de los hombres. Cuando la distancia se expresó en unidades intrínsecas, es decir, como el cociente de la distancia del bloque sobre la longitud del brazo de cada participante, hombres y mujeres distribuyeron sus modos de acción al alcanzar el bloque de manera equiparable a lo largo de las diferentes distancias. No obstante, a la distancia $\pi 1.1$ se observaron diferencias en la ejecución entre hombres y mujeres. La elección del modo de acción cambió en puntos críticos en donde la relación entre la distancia del bloque y la longitud del brazo indujo la aparición de un modo de acción diferente. La elección del modo de acción varió más en y a distancias alrededor del límite crítico absoluto del modo de acción sólo con el brazo. Estos hallazgos sugieren que
\end{abstract}

Enviar correspondencia al primer autor: angel.jimenez@cuci.udg.mx, Centro de Investigación en Conducta y Cognición Comparada, Centro Universitario de la Ciénega, Universidad de Guadalajara, Av. Universidad 1115, C.P. 47820, Ocotlán, Jalisco, México

Los autores agradecen a Ofelia Citlalli López por su asistencia en la revisión de los videos y a Rosalío Ávila y Lilia Prado por facilitarnos un antropómetro para el registro de las dimensiones corporales. Partes de este trabajo fueron presentados en el XXII Congreso Mexicano de Análisis de la Conducta. Guanajuato, México. Noviembre de 2012, y en la 34 Reunión Anual de la Society for the Quantitative Analyses of Behavior (SQAB). Denver, Colorado, EUA. Mayo de 2011. 
la relación entre la escala corporal del sujeto comportante, sus restricciones biomecánicas, y la escala del ambiente contribuyen a la elección del modo de acción.

Palabras clave: Posibilitador de acción, métrica intrínseca, modo de acción, alcanzar con el brazo, topografía de la conducta, humanos.

\begin{abstract}
Two groups of participants, men and women, reached in seated position for a plastic block that was presented on a table at different distances. When the distance was expressed in extrinsic units (centimeters), women changed their modes of action for reaching for the block at closer distances than men because their body sizes were smaller than those of men. When the distance was expressed in intrinsic units; that is, as the ratio between the distance of the block and the arm length of each participant, both men and women allocated their modes of action to reach for the block in a comparable way along the different distances. However, at the $\pi$ distance 1.1 differences were observed in the performance of men and women. The choice of mode of action shifted in critical points where the relationship between the distance of the block and arm length induced the appearance of a different mode of action. The choice of the mode of action varied more at and at distances around the absolute critical boundary of the arm-only mode of action. These findings suggest that the relation between the subject's body scale, his or her biomechanical restrictions, and the scale of the environment, contributes to the choice of mode of action.

Keywords: Affordance, intrinsic metric, mode of action, arm reaching, behavior topography, humans.

Un 'posibilitador de acción' o affordance (Gibson, 1979) es una propiedad de una superficie del ambiente que genera oportunidades de acción para un organismo. Por ejemplo, si una superficie es horizontal (en lugar de inclinada), plana (en vez de cóncava o convexa), suficientemente extensa (relativa al tamaño del organismo), y si su sustancia es rígida (relativa al peso del organismo), entonces esta superficie posibilita acciones como caminar, correr o saltar (Gibson, 1979). De esta forma, un posibilitador de acción es un arreglo del ambiente que sostiene modos de acción específicos en un organismo. Un modo de acción se define como una organización estable y cualitativamente distinta de la musculatura que emerge cuando un organismo ejecuta una respuesta motora (Mark et al., 1997).

La noción de posibilitador de acción, así como su correspondiente descripción conductual referida con el concepto de modo de acción se originaron en el campo de la psicología ecológica (Gibson, 1966, 1979). No obstante, diversos autores han señalado la compatibilidad e incluso complementariedad teórica que existe entre la
\end{abstract}


psicología ecológica y el análisis experimental de la conducta al definirse como psicologías sustentadas en las relaciones que los organismos establecen con el ambiente (Cabrera, Covarrubias, \& Jiménez, 2009; Costall, 1984; Covarrubias, Guzmán, Cabrera, \& Jiménez, 2011; Morris, 2009; Turvey, Solomon, \& Burton, 1989). Particularmente, los posibilitadores de acción han sido vinculados con aspectos del control de estímulos (Catania, 1992; Cabrera, Jiménez, Covarrubias, \& Villanueva, 2013), con propiedades de control contextual (Rosas, Todd, \& Bouton, 2013), y como condiciones iniciales para el aprendizaje (Timberlake, 1993). Por su parte, los modos de acción que emergen a partir de dichos posibilitadores de acción se han vinculado con las topografías de la conducta o aspectos cualitativos de la morfología de la respuesta que emergen ante ciertas configuraciones del ambiente (para una revisión véase Cabrera, Covarrubias, \& Jiménez, 2013).

En sujetos humanos la percepción de los posibilitadores de acción puede evaluarse presentando alguna configuración de estímulos y registrando los modos de acción o topografías con los que la tarea se ejecuta; o bien, pidiendo a los participantes describir si les es posible o no efectuar alguna acción ante los estímulos que el experimentador les presenta.

En un estudio pionero, Warren (1984) presentó a adultos jóvenes fotografías de escalones que tenían diferentes alturas para evaluar la estimación que los participantes hacían con respecto a si los escalones posibilitaban la acción de subirlos utilizando un modo de acción que involucraba emplear exclusivamente las piernas. Con el propósito de caracterizar formalmente la noción de posibilitador de acción, Warren propuso un sistema de medición intrínseco para describir el ajuste del organismo al ambiente. Para ello tomó una propiedad del organismo (la longitud de las piernas de los participantes) como un estándar para medir una propiedad ambiental (la altura del escalón). Si las propiedades del ambiente y del organismo se miden con las mismas unidades y se expresan como una razón o cociente, las unidades se cancelan y el resultado es un número sin unidades o sin dimensiones que expresa un ajuste particular entre una propiedad de un organismo $(\mathrm{O})$ y una propiedad del ambiente $(\mathrm{A})$, al resultado de este cociente Warren lo llamó número $\pi$ :

$\pi=\mathrm{A} / \mathrm{O}$

Cuando las estimaciones de los participantes se expresaron en métrica extrínseca o unidades arbitrarias (i.e., en pulgadas), Warren (1984) encontró que la estimación acerca de la altura del escalón que era 'subible' fue distinta para cada participante dependiendo de la longitud de sus piernas. Los participantes altos estimaron que podían subir escalones más altos que los participantes de estatura baja. No obstante, cuando la altura del escalón se expresó en unidades intrínsecas (Ecuación 1), las 
diferencias entre las estimaciones de los participantes desaparecieron. Los participantes juzgaron que podían subir el escalón usando sólo las piernas cuando éste tuvo una altura máxima equivalente al $88 \%$ de la longitud de sus propias piernas. Cuando la altura del escalón excedió este límite, los participantes juzgaron que no podían subir el escalón usando un modo de acción que involucrara sólo las piernas. Por consiguiente, los participantes juzgaron que un modo de acción distinto era necesario para subir el escalón, uno que involucrara además de las piernas, las manos y los antebrazos (Warren, 1984). Al punto máximo donde los participantes estimaron que les era posible subir el escalón usando un modo de acción bípedo y que más allá de éste necesitaban cambiar a un modo de acción cuadrúpedo, Warren (1984) lo llamó límite crítico absoluto, el cual se refiere a la magnitud de una propiedad ambiental que coincide con el límite de la capacidad física de un organismo para ejecutar un modo de acción particular. Los resultados de Warren sugieren que los juicios acerca de los límites críticos absolutos de los modos de acción están determinados por las restricciones que imponen las propias características antropométricas del individuo. De esta manera, la métrica intrínseca permite identificar que el límite crítico absoluto es el mismo para todos los individuos porque es una proporción invariante de la escala corporal del individuo (Warren, 1995).

Por otra parte, evaluar la percepción de posibilitadores de acción según los modos de acción consiste en registrar el patrón de la conducta motora desplegada al momento de ejecutar una acción. Diversos estudios han extendido la generalidad de los hallazgos de Warren (1984) a evaluaciones de modos de acción en tareas como asir objetos con la mano (Cesari \& Newell, 1999, 2000; Newell, McDonald, \& Baillargeon, 1993), atravesar aberturas caminando (Chang, Wade, \& Stoffregen, 2009; Warren \& Whang, 1987) y alcanzar objetos con el brazo (e.g., Mark et al., 1997). Esta última conducta tiene particular relevancia debido a que una proporción considerable de las acciones motoras que el humano lleva a cabo implican el movimiento de los brazos no sólo para alcanzar, desplazar o depositar objetos, sino también para oprimir botones y accionar palancas. Para determinar si un objeto posibilita la acción de alcanzarlo con el brazo, es necesario analizar las propiedades del ambiente conductualmente relevantes en relación a las capacidades de acción del humano, en esta situación una de las propiedades relevantes del ambiente es la distancia del objeto a alcanzar.

Reconociendo esto, Mark et al. (1997) variaron la distancia a la que se colocó un bloque de plástico sobre una mesa que adultos jóvenes tenían que alcanzar en posición sedente. Mark y colaboradores definieron las distancias en métrica intrínseca (Ecuación 1) y registraron la frecuencia de uso en cada distancia de un modo de acción al que llamaron 'sólo con el brazo'. Este modo de acción consistió en alcanzar el bloque estirando exclusivamente el brazo, sin involucrar otras partes del cuerpo 
como rotar o inclinar el tronco. Los participantes usaron este modo de acción casi de manera exclusiva a distancias inferiores a la longitud de su propio brazo (i.e., a distancias inferiores al límite crítico absoluto del modo de acción sólo con el brazo). De manera interesante, a partir de la distancia $\pi=0.95$ (equivalente al $95 \%$ de la longitud del propio brazo), los participantes dejaron de preferir el modo de acción sólo con el brazo aunque aún les era posible utilizarlo e introdujeron modos de acción que involucraban rotar el tronco o flexionarlo hacia adelante, aunque no describieron la distribución de estos últimos modos de acción.

En un estudio posterior, Gardner, Mark, Ward y Edkins (2001) emplearon un procedimiento similar al reportado por Mark et al. (1997), con la innovación que definieron las distancias en términos del alcance máximo posible de cada participante al extender el brazo y flexionar el tronco hacia adelante sin levantarse del asiento (i.e., modo de acción con el brazo y tronco), mientras que en el estudio de Mark et al. (1997) las distancias se definieron a partir de la longitud del brazo de cada participante. Esto permitió a Gardner et al. (2001) evaluar un rango de distancias más lejanas que en el estudio de Mark et al. (1997). En las distancias más cercanas, Gardner encontró que los participantes emplearon en casi todos los ensayos el modo de acción con el brazo y tronco. En las distancias más lejanas, los participantes cambiaron a un modo de acción que implicó ponerse de pie para alcanzar el objeto. Estos hallazgos junto con los de Mark et al. (1997) son consistentes con la noción de posibilitador de acción (Gibson, 1979) formalizada en los números $\pi$ (Warren, 1984), ya que ésta predice que cuando varía la relación entre las propiedades dimensionales del organismo y del ambiente, las posibilidades de acción del organismo cambian y diferentes características cualitativas de la conducta (i.e., modos de acción) emergen (Warren, 2006). De esta forma, si la distancia del bloque supera la longitud del brazo del participante necesariamente aparecerá un nuevo modo de acción que posibilite la acción de alcance (Mark et al., 1997). Si la distancia del bloque incrementa aún más, emergerán otros modos de acción que posibiliten efectuar el alcance (Gardner et al., 2001). Estos hallazgos muestran que los modos de acción se despliegan de manera ordenada como función de la relación entre las métricas corporal y ambiental.

La métrica intrínseca o formalización del posibilitador de acción, tal como la propuso Warren (1984, 1995), describe los límites críticos absolutos de los diferentes modos de acción tomando en cuenta las propiedades dimensionales o geométricas del organismo en relación a la escala ambiental, por lo que predeciría que el sexo de los participantes no influye en la elección de los modos de acción. Tal vez por esta razón, Mark et al. (1997) no reportaron el sexo de sus participantes y Gardner et al. (2001) basaron sus conclusiones en promedios en los que combinaron los datos de hombres y mujeres. Sin embargo, algunos estudios han encontrado que variables biomecánicas (i.e., fuerza muscular y flexibilidad articular) influyen en la elección de 
los modos de acción. Por ejemplo, Konczak, Meeuwsen y Cress (1992) encontraron que adultos mayores reportaron la necesidad de cambiar de un modo de acción bípedo a un modo de acción cuadrúpedo con escalones de altura más baja que adultos jóvenes. Por otro lado, estudios conducidos en el área de la ergonomía han encontrado diferencias en la flexibilidad de algunas articulaciones de la parte superior del cuerpo entre hombres y mujeres. Por ejemplo, se ha documentado mayor flexibilidad al realizar movimientos de pronación del antebrazo y aducción de la muñeca (Doriot \& Wang, 2006), así como mayor capacidad de abducción del hombro y flexión del codo entre mujeres que entre hombres (Chaffin, Faraway, Zhang, \& Woolley, 2000). Por su parte, los hombres muestran mayor amplitud de movimiento que las mujeres al extender, flexionar lateralmente y rotar axialmente el tronco (Chateauroux \& Wang, 2008; Doriot \& Wang, 2006).

Choi y Mark (2004) registraron la fuerza del bíceps de hombres y mujeres jóvenes en un experimento en el que variaron el peso de objetos que colocaron sobre una mesa a diferentes distancias, definidas en métrica intrínseca, para que en ensayos sucesivos los participantes los alcanzaran en posición sedente. La fuerza de los hombres fue mayor que la de las mujeres y al incrementar el peso del objeto a alcanzar, hombres y mujeres cambiaron sus modos de acción a distancias cada vez más cercanas. No obstante, con los objetos más pesados las mujeres cambiaron sus modos de acción de manera más temprana que los hombres. Estos resultados sugieren que las diferencias encontradas en la distribución de los modos de acción entre hombres y mujeres fueron influidas por la fuerza muscular. Esto es importante porque implicaría que las posibilidades de acción del ambiente no sólo son función de las dimensiones del organismo, sino también de sus capacidades biomecánicas (véase Chemero, 2009).

Ya que evidencia previa sugiere que las diferencias en las restricciones biomecánicas entre hombres y mujeres podrían afectar los límites críticos absolutos de los modos de acción en tareas de alcance con el brazo, el presente estudio tuvo como primer propósito analizar en un grupo de hombres y en un grupo de mujeres la probabilidad de desplegar diferentes modos de acción en función de la distancia de un objeto, sin manipular su peso. Con tal fin implementamos una situación experimental similar a la que empleó Mark et al. (1997) en la que participantes sentados alcanzaron un bloque de plástico colocado sobre una mesa a diferentes distancias definidas en métrica intrínseca (Ecuación 1). Si la distribución de los modos de acción fuera diferente entre hombres y mujeres, esto sugeriría que variables biomecánicas como la fuerza y la flexibilidad articular contribuyen en la elección de los modos de acción en tareas de alcance con el brazo y resaltaría la necesidad de analizar por separado la ejecución de hombres y mujeres en los estudios de posibilitadores de acción.

Un segundo objetivo consistió en ofrecer una descripción de la distribución de los modos de acción desplegados en el rango de distancias evaluadas por Mark 
et al. (1997). Aunque en estudios previos se describen distribuciones de modos de acción, los rangos de distancias evaluados en éstos son diferentes que en el estudio de Mark et al. (1997). Por ejemplo, Gardner et al. (2001) describieron la transición del modo de acción con el brazo y tronco al modo de acción alcance de pie. Por su parte, Choi y Mark (2004) describieron la transición del modo de acción con el brazo y hombro al modo de acción con el brazo y tronco. Sin embargo, en la literatura no hay una descripción de la transición del modo de acción sólo con el brazo al modo de acción con el brazo y hombro, descripción que puede efectuarse evaluando los modos de acción en el rango de distancias examinadas por Mark et al. (1997). Esto es relevante en el contexto de la generación de evidencia empírica que muestre el valor descriptivo de la métrica intrínseca en tareas motoras.

\section{Método}

\section{Participantes}

Dieciséis estudiantes de licenciatura, ocho hombres (participantes 1 a 8) y ocho mujeres (participantes 9 a 16), con un rango de edad entre los 18 y 25 años. Todos los participantes eran diestros y fueron evaluados de manera individual.

\section{Aparatos y situación experimental}

El experimento se llevó a cabo en una habitación de $5 \times 4 \mathrm{~m}$. Los participantes se sentaron en una silla de altura ajustable a la que se le retiró el respaldo, frente a una mesa de altura ajustable de $70 \mathrm{~cm}$ de largo por $45 \mathrm{~cm}$ de ancho (ver Figura 2). Sobre la superficie de la mesa se presentó a diferentes distancias un bloque de plástico de color rojo de $3 \mathrm{~cm}$ de largo, $1.5 \mathrm{~cm}$ de ancho y $2 \mathrm{~cm}$ de alto. Se utilizó una videocámara Sony, modelo Handycam, montada en un tripié para grabar la sesión experimental desde el costado derecho del participante, una regla $\mathrm{T}$, una computadora portátil Hewlett Packard, una impresora Hewlett Packard, hojas de registro, un banco de asiento plano y altura ajustable, y un antropómetro tipo Martin.

\section{Mediciones antropométricas}

Antes de iniciar la sesión experimental se pidió a cada participante sentarse sobre el banco de asiento plano y altura ajustable. Una vez que el participante estaba sentado, el experimentador ajustó el banco a una altura en la que las piernas formaban un ángulo recto con los muslos, y los pies descansaban completamente en el piso; se pidió al participante mantener el tronco erecto y poner las manos sobre los muslos 
(ver Figura 1). Estando el participante en esta postura se midieron con el antropómetro dos dimensiones corporales: 1) la altura poplítea, que se definió como la distancia entre el piso y el hueco poplíteo (hueco que se forma al sentarse en el vértice que forman la pierna y el muslo) con el participante en postura sedente (ver Figura 1), y 2) la altura al hombro, definida como la distancia comprendida desde la superficie del asiento hasta el acromion (hueso que forma la cresta superior del hombro) con el participante en postura sedente (ver Figura 1). Estas mediciones permitieron ajustar la altura de la silla que se usó en el experimento a la altura poplítea del participante y ajustar la altura de la mesa que se usó en el experimento a la mitad de la distancia entre la altura poplítea y la altura al hombro del participante.

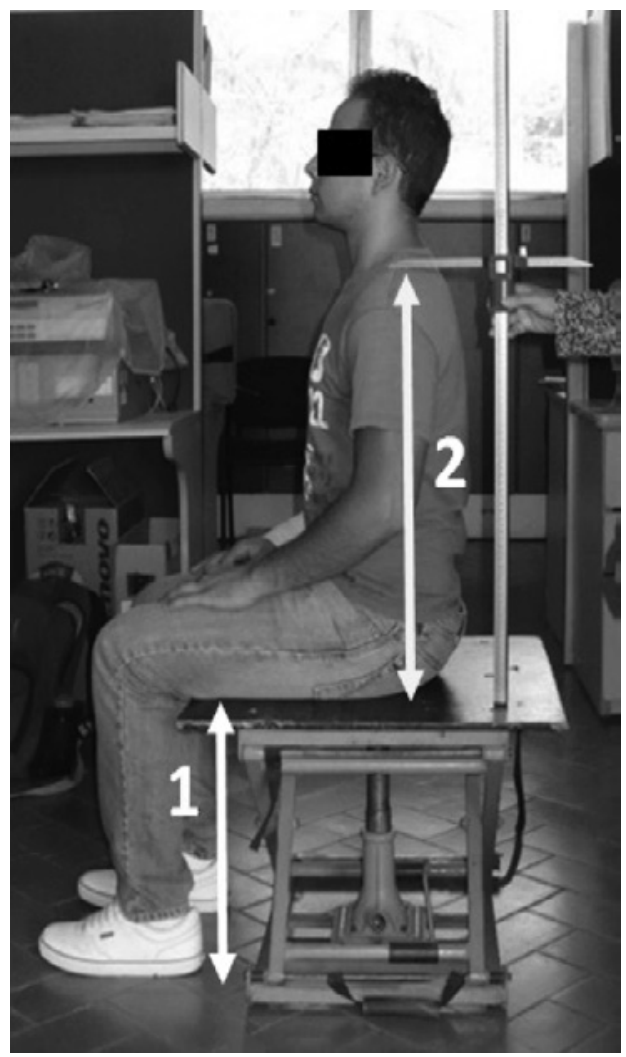

Figura 1. Mediciones antropométricas obtenidas. El número (1) indica la definición de la altura poplítea. El número (2) muestra la definición de la altura al hombro. Véase el texto para más detalles. 
Las alturas de la silla y de la mesa se ajustaron a las dimensiones corporales de cada participante para que la altura de la mesa fuera proporcionalmente igual para todos los participantes. Esto es importante porque la altura de la mesa afecta el rango de alcance del brazo al modificar el ángulo del hombro (i.e., el ángulo formado por el codo, el hombro [acromion] y la cadera). Mientras más baja es la altura de la mesa, disminuye la distancia a la que un participante puede alcanzar un objeto utilizando sólo el brazo (Carello, Grosofsky, Reichel, Solomon, \& Turvey, 1989; Chateauroux \& Wang, 2008). Al ajustar la altura de la silla y de la mesa a las dimensiones corporales de cada participante logramos que el hombro de todos los participantes se encontrara en un ángulo similar al momento de llevarse a cabo las acciones de alcance, de modo que los datos de todos los participantes fueran comparables entre sí.

\section{Procedimiento}

Los participantes fueron evaluados en una sola sesión experimental, misma que tuvo una duración aproximada de 35 minutos. A cada participante se le pidió autorización para ser grabado en video y se le informó que estaba participando en un estudio acerca de cómo nuestra percepción del mundo guía nuestras acciones, y que en particular estábamos interesados en comprender cómo la distancia de los objetos afecta la manera en que la gente los alcanza. Con las alturas de la silla y de la mesa ajustadas a las dimensiones corporales del participante, se le pidió sentarse en la silla frente a la mesa formando con los muslos un ángulo recto con las piernas y extender el brazo derecho de manera que tocara la superficie de la mesa con la palma de la mano (ver Figura 2).

Con el participante en esa postura, se ajustó la ubicación de la silla hacia atrás o hacia adelante para alinear de manera vertical el codo del participante al borde anterior de la mesa (ver Figura 2). Esta distancia definió la distancia del participante con respecto a la mesa en todos los ensayos. Esta acción permitió asegurarnos que todos los participantes se sentaron a la misma distancia de la mesa relativa a las longitudes de sus brazos.

Estando aún el participante sentado en la misma postura y a la misma distancia de la mesa (Figura 2), con la regla T se midió la distancia entre el borde anterior de la mesa y la punta del dedo pulgar del participante (ver Figura 3). Esta distancia definió la longitud del brazo del participante, la cual se utilizó para calcular las distancias a las que se colocó el bloque de plástico. Esto es, las distancias se definieron en términos de métrica intrínseca o números $\pi$ (Ecuación 1), y con ello se equiparó la distancia del bloque a través de los participantes. En ensayos consecutivos, el bloque de plástico se presentó en orden aleatorio a 11 distancias $\pi$ : 0.70, 0.75, 0.80, 0.85, 0.90, $0.95,1.00,1.10,1.20,1.30$ y 1.40. Cada una de las distancias se evaluó 4 veces, por 
lo que en total el experimento consistió en 44 ensayos. Cada distancia correspondió a una proporción de la longitud del brazo. Por ejemplo, si la longitud del brazo de un participante era de $40 \mathrm{~cm}$, ésta distancia correspondió a $\pi=1.00$. Para este participante la distancia $\pi=1.10$ correspondió a $44 \mathrm{~cm}$ (i.e., $10 \%$ más que el límite crítico absoluto del alcance de su brazo).

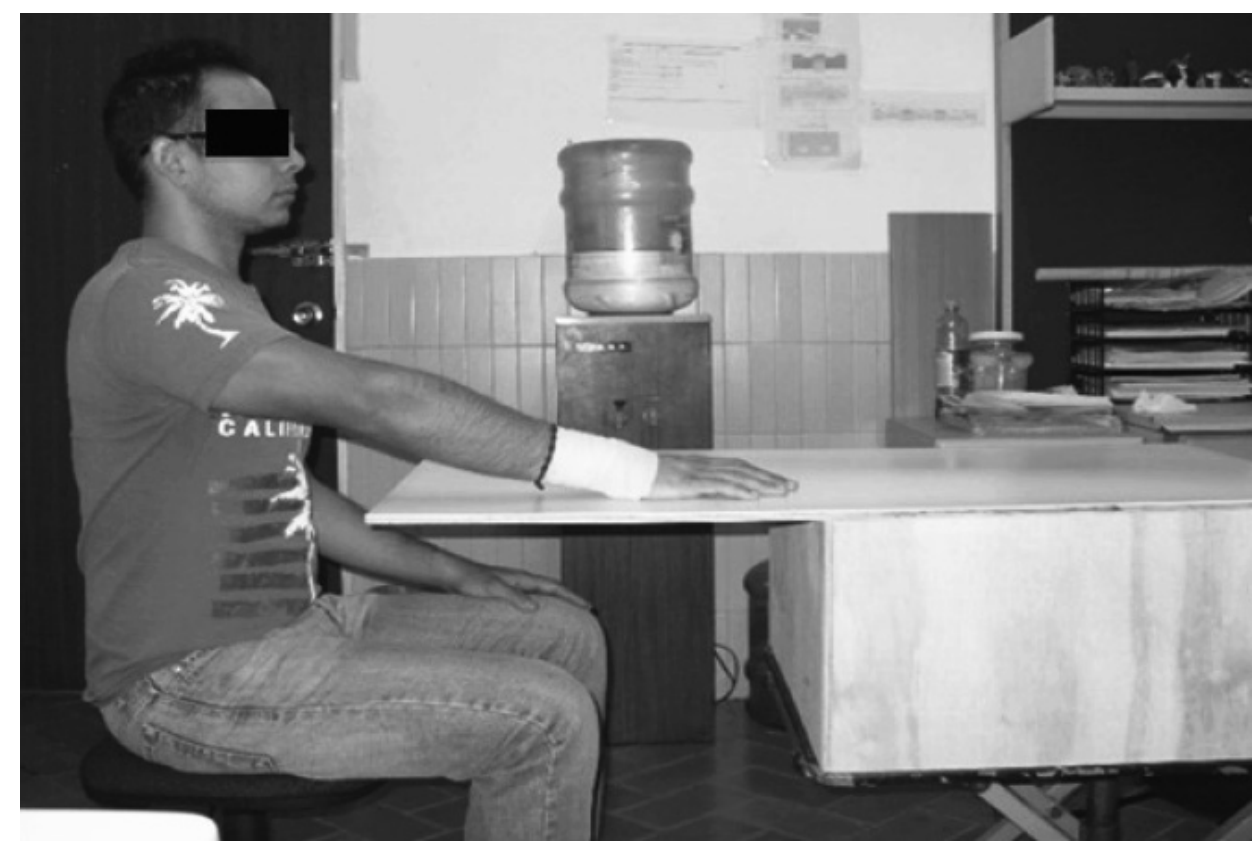

Figura 2. Situación experimental y postura en la que se midió la longitud del brazo de los participantes. Véanse detalles en el texto.

Una vez definidas las distancias de acuerdo a la longitud del brazo del participante, a continuación se le daban las siguientes instrucciones:

"Voy a colocar este bloque de plástico a varias distancias sobre la mesa. Tu tarea va a consistir en alcanzarlo con el brazo derecho de la manera que quieras, pero sin levantarte ni mover el asiento. Vas a agarrar el bloque usando los dedos índice y pulgar. Vamos a hacer varios ensayos, entre un ensayo y otro te pido que por favor cierres los ojos y mantengas las manos sobre tus muslos para que nos permitas acomodar el bloque a una distancia nueva. Yo te indicaré en qué momento los puedes abrir ¿Tienes alguna duda?". 


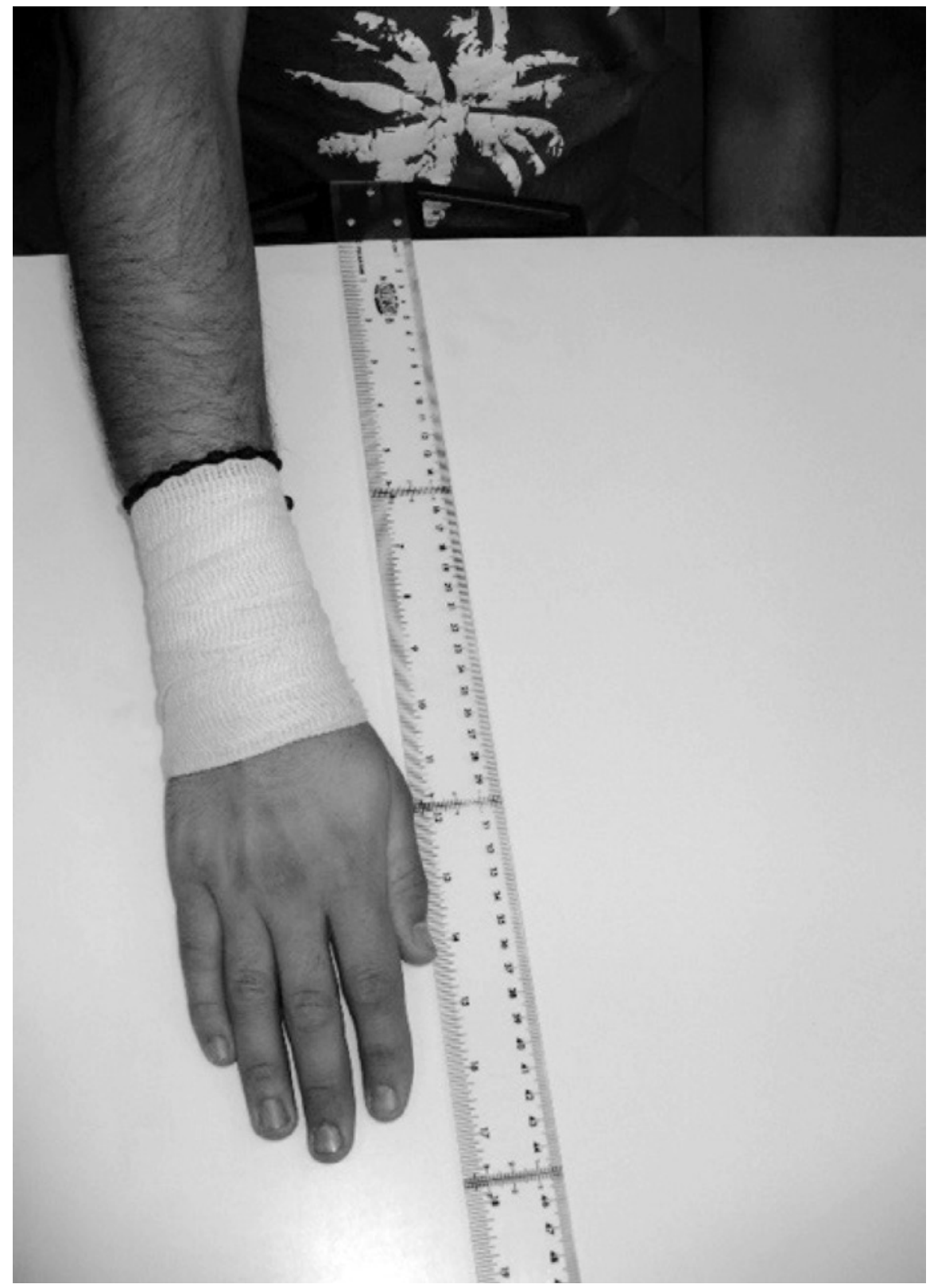

Figura 3. Medición de la longitud del brazo. Véanse detalles en el texto. 
Si el participante tenía dudas, en ese momento se le respondían. Cada ensayo iniciaba pidiendo al participante que cerrara los ojos y mientras el participante tenía los ojos cerrados uno de los experimentadores acomodaba el bloque a la distancia que correspondía para ese ensayo. A continuación se le pedía que abriera los ojos y que alcanzara el bloque, con lo cual terminaba el ensayo. En todos los ensayos el bloque se colocó alineado al brazo derecho del participante.

\section{Sesiones piloto}

Antes de las sesiones experimentales, se corrieron 5 sesiones piloto con participantes que no formaron parte de la muestra experimental con la finalidad de poner a prueba el procedimiento y hacer correcciones en el mismo. La revisión de los videos de estas sesiones piloto nos permitió hacer una taxonomía de los modos de acción usados por los participantes al alcanzar el bloque en el rango de distancias evaluadas (de 23 a $54 \mathrm{~cm}$ ). A partir de este análisis se identificó de manera consistente que en cada ensayo los participantes utilizaron uno de los siguientes tres modos de acción: a) modo de acción "sólo con el brazo", consistió en alcanzar el bloque extendiendo el brazo, la mano y los dedos hacia adelante, sin rotar ni flexionar el tronco hacia adelante (Mark et al., 1997); b) modo de acción "con el brazo y hombro", consistió en extender el brazo, mano y dedos hacia adelante rotando el tronco sin flexionarlo hacia adelante (Gardner et al., 2001). Esta rotación del tronco desplazaba el acromion derecho hacia adelante y permitía alcanzar un poco más lejos que con el modo de acción sólo con el brazo; y c) modo de acción "con el brazo y tronco", consistió en extender el brazo, mano y dedos hacia adelante flexionando el tronco al nivel de la cintura. Esta flexión desplazaba ambos hombros hacia adelante, permitiendo alcanzar más lejos que con el modo de acción con el brazo y hombro.

\section{Análisis de datos}

Los videos de las sesiones experimentales fueron evaluados por dos jueces de manera independiente, la tarea de los jueces consistió en determinar el modo de acción usado por cada participante en cada ensayo. Las evaluaciones de los jueces coincidieron en el $89 \%$ de los ensayos, los videos de los ensayos restantes fueron revisados de nuevo por los dos jueces de manera simultánea en la misma computadora hasta llegar a un acuerdo. Antes del inicio de los ensayos y con la intención de facilitar la distinción entre el modo de acción sólo con el brazo y el modo de acción con el brazo y hombro, pegamos una etiqueta de color amarillo fosforescente (de $2 \times 2 \mathrm{~cm}$ ) sobre el omóplato derecho de los participantes. Al revisar los videos, si el participante rotaba el tronco la etiqueta se hacía visible, si no rotaba el tronco la etiqueta no se veía en el video. 


\section{Resultados}

La Figura 4 muestra el promedio de la proporción de ensayos en que hombres y mujeres usaron los diferentes modos de acción, como función de las distancias a las que se colocó el bloque expresadas en centímetros. Los cuadros llenos y vacíos representan la distribución del modo de acción sólo con el brazo para hombres y mujeres, respectivamente. La línea negra gruesa y la línea quebrada simbolizan la distribución del modo de acción con el brazo y hombro de hombres y mujeres, respectivamente. La distribución del modo de acción con el brazo y tronco se representa con los triángulos llenos para los hombres y vacíos para las mujeres. La Figura 4 revela que para los tres modos de acción, las curvas de ambos grupos tienen una forma similar. Sin embargo, las tres curvas de los hombres se encuentran hacia la derecha de las tres curvas correspondientes a las mujeres, lo cual significa que los hombres cambiaron sus modos de acción a distancias más lejanas que las mujeres debido a que éstos tenían brazos más largos.

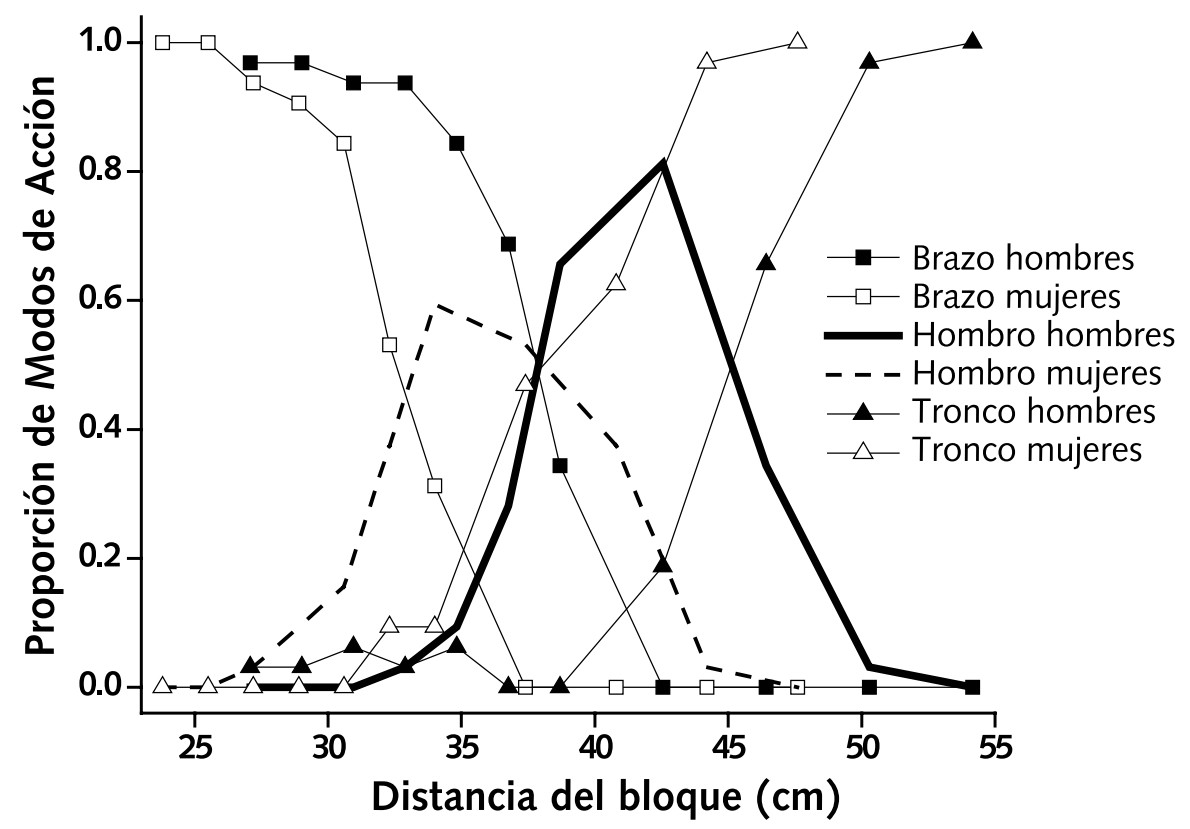

Figura 4. Proporción del uso de los modos de acción sólo con el brazo, con el brazo y hombro, y con el brazo y tronco en función de la distancia del bloque expresada en unidades extrínsecas (centímetros). La Figura muestra los datos promedio de los dos grupos, hombres y mujeres. 
En la Figura 5, el eje de la ordenada representa la proporción de ensayos en que los participantes utilizaron los diferentes modos de acción. El eje de la abscisa representa el cociente de la distancia del bloque sobre la longitud del brazo, el cual corresponde a la expresión de las distancias a las que se colocó el bloque en métrica intrínseca o números $\pi$ (Ecuación 1). La línea vertical alineada a $\pi=1.0$ en el eje horizontal indica el límite crítico absoluto del modo de acción sólo con el brazo o distancia igual a la longitud del brazo del participante. La Figura 5 muestra los resultados promedio de los hombres y las mujeres (véanse los Apéndices A y B para revisar los datos de los individuos). En la Figura 5 se observa que las curvas de los hombres y las curvas de las mujeres tienden a encimarse, lo cual muestra que la distribución de los modos de acción entre hombres y mujeres fue similar cuando la distancia del bloque se expresó en unidades intrínsecas.

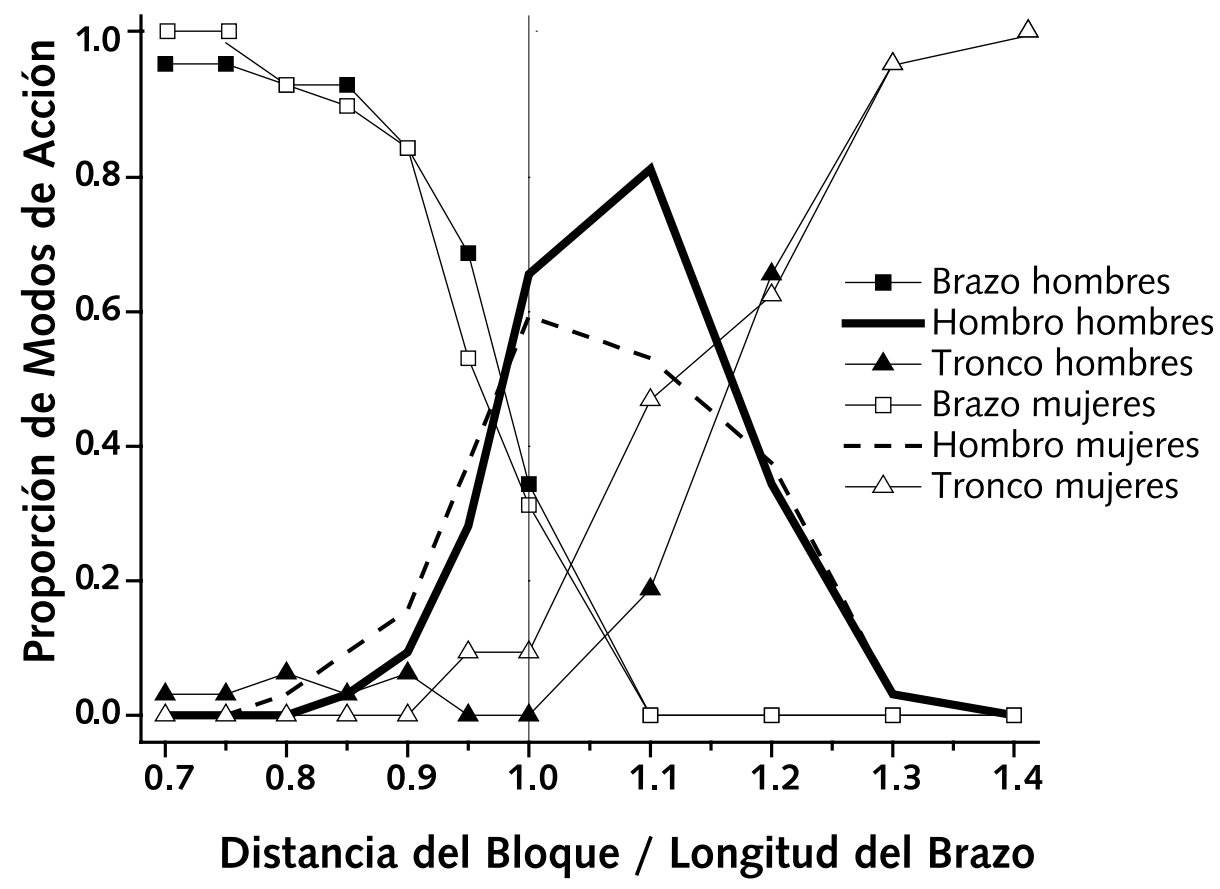

Figura 5. Proporción del uso de los modos de acción sólo con el brazo, con el brazo y hombro, y con el brazo y tronco en función de la distancia expresada en unidades intrínsecas como la razón entre distancia del bloque sobre la longitud del brazo. La Figura muestra los datos promedio de los dos grupos, hombres y mujeres. 
Cuando el bloque se colocó a distancias inferiores al límite crítico absoluto del modo de acción sólo con el brazo (i.e., de $\pi=0.7$ a $\pi=0.85$ ), hombres y mujeres usaron el modo de acción sólo con el brazo en una proporción mayor al 0.9 de los ensayos. Hasta la distancia $\pi=0.95$ la preferencia de hombres y mujeres favoreció al modo de acción sólo con el brazo (proporción mayor al 0.5 de los ensayos). Al incrementar la distancia del bloque la preferencia por este modo de acción disminuyó, de manera tal que a la distancia $\pi=1.0$, hombres y mujeres usaron este modo de acción en una proporción de 0.34 y 0.31 de los ensayos, respectivamente. Este resultado muestra que los participantes dejaron de usar el modo de acción sólo con el brazo a una distancia ( $\pi=1.0$ ) en la que aún les era posible utilizarlo. A partir de la distancia $\pi=1.1$ el modo de acción sólo con el brazo no ocurrió más ya que no era físicamente posible llevarlo a cabo, debido a que la distancia del bloque excedía la longitud del brazo.

La Figura 5 revela que al incrementar la distancia del bloque, incrementó la preferencia por el modo de acción con el brazo y hombro (al descender la preferencia por el modo de acción sólo con el brazo). Para hombres y mujeres, la preferencia favoreció a este modo de acción a las distancias $\pi=1.0$ y 1.1 (proporciones mayores a 0.5). La distancia en la que los hombres mostraron una mayor preferencia por el modo de acción con el brazo y hombro fue en $\pi=1.1$ (proporción de 0.81 de los ensayos). La distancia a la que las mujeres usaron más el modo de acción con el brazo y hombro fue en $\pi=1.0$ (proporción de 0.59 de los ensayos). Para ambos grupos, a distancias más lejanas de la distancia a la que la preferencia de este modo de acción fue extrema, la preferencia por el modo de acción con el brazo y hombro disminuyó, de manera que a la distancia $\pi=1.4$ este modo de acción ya no ocurrió, posiblemente debido a que a esta distancia ya no era posible para los participantes alcanzar con el modo de acción con el brazo y hombro.

Con respecto al modo de acción con el brazo y tronco, a las distancias más cercanas a las que se colocó el bloque (de $\pi=0.7$ a $\pi=1.0$ ) hombres y mujeres usaron este modo de acción en una proporción inferior al 0.1 de los ensayos. Más aún, la Figura 5 muestra que en algunas de estas distancias, la proporción de uso fue de 0.0, debido a que a esas distancias los participantes usaban los modos de acción sólo con el brazo y con el brazo y hombro. La preferencia de hombres y mujeres comenzó a favorecer el modo de acción con el brazo y tronco a partir de la distancia $\pi=1.2$ (proporción superior al 0.6 de los ensayos). A la distancia $\pi=1.4$, la preferencia de hombres y mujeres por el modo de acción con el brazo y tronco fue exclusiva.

Aunque la Figura 5 muestra que las curvas de los hombres y de las mujeres tienden a encimarse, puede verse que los hombres usaron más que las mujeres el modo de acción con el brazo y hombro a la distancia $\pi=1.1$ ( 0.81 y 0.53 de los ensayos, respectivamente). Esto se debió a que a esta distancia, las mujeres usaron más que los 
hombres el modo de acción con el brazo y tronco ( 0.47 y 0.19 de los ensayos, respectivamente). En otras palabras, las mujeres comenzaron a usar más el modo de acción con el brazo y tronco a una distancia en que los hombres aún usaban mayoritariamente el modo de acción con el brazo y hombro.

Con el propósito de conocer el grado en que las distribuciones de los tres modos de acción variaron a lo largo de las diferentes distancias, se calculó la desviación estándar. La distribución de la desviación estándar de los hombres y las mujeres se muestra en la Figura 6 como función de la distancia expresada en números $\pi$ (Ecuación 1) para cada uno de los tres modos de acción. Se encontró que la desviación estándar tuvo una distribución similar para los tres modos de acción. Para el modo de acción sólo con el brazo, los valores de la desviación estándar más altos ocurrieron a distancias entre $\pi=0.9$ y $\pi=1.0$. Para el modo de acción con el brazo y hombro las desviaciones estándar más altas se registraron a distancias $\pi$ entre 0.9 y 1.2. En lo referente al modo de acción con el brazo y tronco, las mayores desviaciones estándar se presentaron entre $\pi=1.0$ y $\pi=1.2$. En general, las tres gráficas de la Figura 6 muestran que los valores más altos de la desviación estándar se distribuyeron a distancias alrededor del límite crítico absoluto del modo de acción sólo con el brazo y que las desviaciones estándar fueron mayores entre las mujeres que entre los hombres.

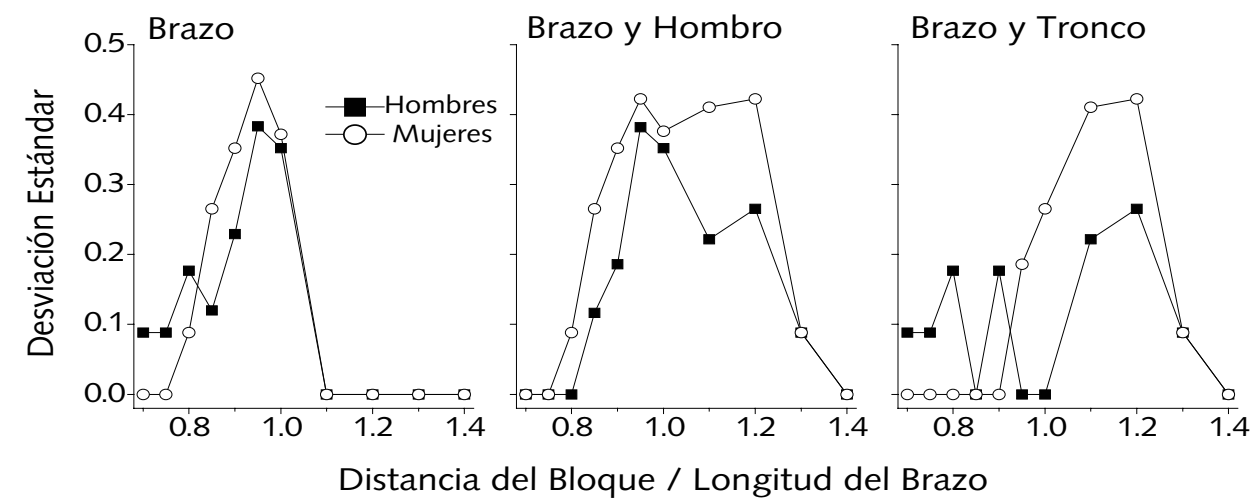

Figura 6. Distribución de la desviación estándar de los tres modos de acción como función de la distancia expresada en métrica intrínseca como la razón entre la distancia del bloque sobre la longitud del brazo de los participantes.

\section{Discusión}

Los resultados de este experimento muestran que los modos de acción cambiaron su distribución de manera ordenada como función de la distancia del bloque. Como 
se esperaba, cuando la distancia se expresó en centímetros, una unidad extrínseca o arbitraria, se observó que las mujeres cambiaron sus modos de acción a distancias más cercanas que los hombres debido a que éstas tenían brazos más cortos que los hombres. No obstante, cuando la distancia se expresó en métrica intrínseca (Ecuación 1), se observó que los modos de acción cambiaron a distancias proporcionalmente similares entre hombres y mujeres. Así, cuando el bloque se ubicó a distancias inferiores al límite crítico absoluto del modo de acción sólo con el brazo, la elección de los hombres y las mujeres favoreció al modo de acción sólo con el brazo. Cuando el bloque se alcanzó a distancias alrededor del límite crítico absoluto del modo de acción sólo con el brazo, en la mayoría de los ensayos los hombres y las mujeres eligieron el modo de acción con el brazo y hombro. Finalmente, con el bloque a distancias que superaban el límite crítico absoluto del modo de acción sólo con el brazo emergió para hombres y mujeres el modo de acción con el brazo y tronco.

Estos resultados revelan que la elección del modo de acción dependió de la relación recíproca entre las dimensiones del ambiente (la distancia del bloque) y las dimensiones del participante (la longitud del brazo), induciendo cambios en la forma de la conducta cuando los participantes llegaban a puntos críticos o límites críticos absolutos de esta relación ambiente-organismo. Lo anterior sugiere que la distancia del bloque, definida en métrica intrínseca, posibilitó la emergencia de distintos modos de acción. De esta manera, la métrica intrínseca permitió identificar las invariancias entre la escala corporal del participante y las propiedades del ambiente requeridas para sostener la conducta de alcanzar un bloque con el brazo. Los presentes hallazgos también sugieren que el concepto de posibilitador de acción (Gibson, 1979) expresado como números $\pi$ (Warren, 1984), permite describir la distribución de diferentes modos de acción, así como dar cuenta de cambios en su morfología.

Los presentes resultados son consistentes con los hallazgos de experimentos que evaluaron acciones de alcance (Choi \& Mark, 2004; Gardner et al., 2001; Mark et al., 1997), acciones prensiles (Cesari \& Newell, 1999, 2000; Lopresti-Goodman, Richardson, Baron, Carello, \& Marsh, 2009; Newell et al., 1993), atravesar aberturas caminando (Chang, Wade, \& Stoffregen, 2009; Warren \& Whang, 1987), y subir escalones (Konczak et al., 1992; Warren, 1984). En conjunto, todos estos estudios proporcionan evidencia de que la elección de los modos de acción depende de una métrica basada en la escala corporal, lo cual constituye a su vez una métrica basada en las capacidades conductuales del organismo definidas por sus propias características estructurales.

Aunque se observó que la distribución de los modos de acción a lo largo de las diferentes distancias a las que se colocó el bloque, expresadas como números $\pi$, fue similar entre hombres y mujeres, se encontró que las mujeres disminuyeron la proporción de uso del modo de acción con el brazo y hombro e incrementaron 
la proporción de uso del modo de acción con el brazo y tronco a una distancia $\pi$ más cercana que los hombres. Dado que la ejecución del modo de acción con el brazo y hombro implica la rotación del tronco, este resultado es consistente con estudios que han mostrado que durante la ejecución de alcances con el brazo las mujeres muestran menor rotación axial del tronco que los hombres (Chateauroux \& Wang, 2008). Por lo tanto, esta diferencia en la ejecución al llevar a cabo alcances con el brazo encontrada entre hombres y mujeres sugiere que las posibilidades de acción del ambiente (el bloque colocado a diferentes distancias) no sólo interactuaron con la antropometría, sino que también fueron influidas por la flexibilidad del tronco de los participantes. Este hallazgo armoniza con los resultados reportados por Choi y Mark (2004) quienes encontraron diferencias en la ejecución de hombres y mujeres en acciones de alcance de objetos de diferente peso. En conjunto, nuestro estudio y el de Choi y Mark (2004) sugieren que las restricciones biomecánicas de la fuerza muscular y flexibilidad articular contribuyen a la definición de los límites críticos absolutos de los modos de acción.

De esta manera, los presentes resultados podrían indicar que a la distancia $\pi=$ 1.1, diferencias biomecánicas entre hombres y mujeres ocasionaron que la distancia del bloque posibilitara modos de acción diferentes para los hombres y las mujeres. Más aún, las diferencias observadas en la ejecución entre individuos cuando la distancia se expresó en métrica intrínseca (véanse los Apéndices A y B) también podrían deberse a diferencias en las capacidades biomecánicas entre los participantes. Estudios futuros sobre posibilitadores de acción podrían registrar variables como la movilidad de la articulación del hombro, y la flexión y rotación del tronco en participantes que realicen acciones de alcance para someter a prueba esta hipótesis. Lo anterior sugiere que el modelo de los números $\pi$ (Ecuación 1) propuesto por Warren (1984, 1995) podría resultar insuficiente para describir cuantitativamente los límites críticos absolutos de los diferentes modos de acción al no considerar la influencia de las restricciones biomecánicas del individuo en la emergencia de los modos de acción.

Entre hombres y mujeres, la preferencia por el modo de acción sólo con el brazo cambió por el modo de acción con el brazo y hombro a la distancia $\pi=1.0$; distancia en la que aún era posible usar el modo de acción sólo con el brazo. Este resultado coincide con el reporte de Mark y colaboradores (1997). Una posible interpretación de este resultado es que cuando un organismo interactúa con una superficie ambiental, éste tiende a cambiar su modo de acción ante dimensiones ambientales menores a su propio límite crítico absoluto porque esto le resulta más económico en términos energéticos. Un experimento que apoya esta hipótesis fue conducido por Warren (1984, Experimento 2), quien registró la tasa cardiaca de adultos jóvenes mientras subían escalones de diferentes alturas y encontró que la altura "óptima" del escalón, esto es, la altura en la que los participantes registraron la menor tasa cardiaca al subir 
los escalones fue de $\pi=0.25$, altura muy inferior al límite crítico absoluto $(\pi=1.0$, una altura equivalente al $88 \%$ de la longitud de las piernas de cada participante) del modo de acción que involucraba subir el escalón sólo con las piernas.

El análisis de las desviaciones estándar mostró que en el límite crítico absoluto del modo de acción sólo con el brazo y a las distancias alrededor de éste, la elección del modo de acción varió más que a distancias lejanas a este límite crítico absoluto. Este hallazgo es consistente con estudios que evaluaron la percepción de posibilitadores de acción mediante la estimación de la 'alcanzabilidad' de una línea en el plano vertical (Cabrera, Jiménez et al., 2013), y pudo deberse a que las distancias más cercanas (de $\pi=0.7$ a $\pi=0.85$ ) constituyeron una región estable en donde se prefirió el uso del modo de acción sólo con el brazo sobre los otros modos de acción, probablemente porque éste modo de acción se asoció con un gasto energético menor (Warren, 1984) o con un mejor ajuste a las posibilidades de acción, generando poca variabilidad en la elección del modo de acción. Por otra parte, en el límite crítico absoluto del modo de acción sólo con el brazo y en distancias cercanas al mismo, la elección del modo de acción se volvió más variable porque estas distancias representaron una región de transición de la conducta en donde el ajuste del organismo al ambiente cambió favoreciendo la emergencia de otro modo de acción. Este hallazgo coincide con estudios sobre coordinación motora que reportan mayor variabilidad de la conducta en las fases de transición de un patrón de coordinación motor a otro (Kelso, 1995; Kelso \& Schöner, 1988).

\section{Posibilitadores de acción y modos de acción en el análisis experimental de la conducta}

Recientemente, Cabrera, Sanabria, Jiménez y Covarrubias (2013) presentaron un estudio con dos especies de roedores en el que variaron la altura de la palanca en nivel operante (i.e., antes de la entrega de reforzamiento). Los resultados mostraron que la tasa de conducta de presionar la palanca fue más alta cuando la altura de la palanca correspondió con la altura de las patas delanteras de ambas especies de roedores, que cuando la palanca se presentó a alturas más bajas o más altas que la altura de las patas delanteras de los roedores. Este hallazgo sugiere que la conducta de presionar la palanca en nivel operante es sensible a las posibilidades de acción del ambiente (i.e., a la relación entre la métrica ambiental y la métrica del organismo). Si esto es cierto, es posible que la tasa de respuestas condicionada instrumentalmente sea afectada por los posibilitadores de acción del ambiente; como por ejemplo, la altura del operando, por lo que estudios futuros podrían poner a prueba esta hipótesis.

La investigación presentada aquí es consistente en términos conceptuales y metodológicos con la visión molar de la conducta (Baum, 1995, 2001, 2002, 2004, 2012) propuesta al interior del análisis experimental de la conducta. Nuestra investigación 
examinó la conducta de alcanzar objetos con el brazo, una conducta molar; esto es, una conducta con extensión temporal. La investigación desarrollada desde esta visión ha descrito la distribución de conductas tanto discretas como molares en dos o más alternativas de respuesta (e.g., Baum \& Davison, 2004; Baum \& Rachlin, 1969; Cabrera \& Aparicio, 2006; Jiménez \& Aparicio, 2009, 2014). De la misma forma, en este estudio se describió la distribución de diferentes modos de acción (conductas molares) a lo largo de las diferentes distancias a las que se colocó un objeto que los participantes tenía que alcanzar, extendiendo con este análisis la generalidad de la visión molar de la conducta a la respuesta motora de alcanzar un objeto con el brazo. En conclusión, el presente escrito intenta ser una contribución para lograr una mayor complementariedad entre la perspectiva ecológica de la psicología y el análisis experimental de la conducta.

\section{Referencias}

Baum, W. M. (1995). Introduction to molar behavior analysis. Mexican Journal of Behavior Analysis, 21, 7-25.

Baum, W. M. (2001). Molar versus molecular as a paradigm clash. Journal of the Experimental Analysis of Behavior, 75, 338-341.

Baum, W. M. (2002). From molecular to molar: a paradigm shift in behavior analysis. Journal of the Experimental Analysis of Behavior, 78, 95-116.

Baum, W. M. (2004). Molar and molecular views of choice. Behavioural Processes, 66, 349-359.

Baum, W. M. (2012). Rethinking reinforcement: allocation, induction, and contingency. Journal of the Experimental Analysis of Behavior, 97, 101-124.

Baum, W. M. \& Davison, M. (2004). Choice in a variable environment: Visit patterns in the dynamics of choice. Journal of the Experimental Analysis of Behavior, 81, 85-127.

Baum, W. M. \& Rachlin, H. (1969). Choice as time allocation. Journal of the Experimental Analysis of Behavior, 12, 861-874.

Cabrera, F. \& Aparicio, C. F. (2006). Travel, sensitivity to reinforcement, and multiple alternatives. Brazilian Journal of Behavior Analysis, 2, 221-234.

Cabrera, F., Covarrubias, P., \& Jiménez, Á. A. (2009). Los sistemas conductuales desde una aproximación ecológica. En J. Varela, F. Cabrera, \& J. J. Irigoyen (Eds.), Estudios sobre comportamiento y aplicaciones: Vol. I (pp. 31-50). México: Universidad de Guadalajara.

Cabrera, F., Covarrubias, P., \& Jiménez, Á. A. (2013). Topografía de la conducta en función de la configuración de las superficies: El caso del nivel operante. En J. J. Irigoyen, F. Cabrera, M. Y. Jiménez, H. Martínez, \& K. F. Acuña (Eds.), Estudios sobre comportamiento y aplicaciones: Vol. III (pp. 48-65). México: Universidad de Sonora. 
Cabrera, F., Jiménez, Á. A., Covarrubias, P., \& Villanueva, S. (2013). Estimando la 'alcanzabilidad' de una línea: una aproximación a la percepción de posibilitadores de acción. Revista Mexicana de Análisis de la Conducta, 39, 62-91.

Cabrera, F., Sanabria, F., Jiménez, Á. A., \& Covarrubias, P. (2013). An affordance analysis of unconditioned lever pressing in rats and hamsters. Behavioural Processes, 92, 36-46.

Carello, C., Grosofsky, A., Reichel, F. D., Solomon, H. Y., \& Turvey, M. T. (1989). Visually perceiving what is reachable. Ecological Psychology, 1, 27-54.

Catania, C. (1992). Learning. New Jersey: Prentice Hall.

Cesari, P. \& Newell, K. M. (1999). The scaling of human grip configurations. Journal of Experimental Psychology: Human Perception and Performance, 25, 927-935.

Cesari, P. \& Newell, K. M. (2000). Body scaling of grip configurations in children aged 6-12 years. Developmental Psychobiology, 36, 301-310.

Costall, A. P. (1984). Are theories of perception necessary? A review of Gibson's the ecological approach to visual perception. Journal of the Experimental Analysis of Behavior, 41, 109-115.

Covarrubias, P., Guzmán, R., Cabrera, F., \& Jiménez, Á. A. (2011). Las superficies ambientales, la velocidad y la aceleración en hámsteres y ratas. En. H. Martínez, J. J. Irigoyen, F. Cabrera, J. Varela, P. Covarrubias, \& Á. A. Jiménez. (Eds.), Estudios sobre comportamiento y aplicaciones: Vol. II (pp. 95-115). México: Universidad de Guadalajara.

Chaffin, D. B., Faraway, J. J., Zhang, X. \& Woolley, C. (2000). Stature, age, and gender effects on reach motion postures. Human Factors, 42, 408-420.

Chang, C.-h., Wade, M. G., \& Stoffregen, T. A. (2009). Perceiving affordances for aperture passage in an environment-person-person system. Journal of Motor Behavior, 41, 495-500.

Chateauroux, E. \& Wang, X. (2008). Effects of age, gender and target location on seated reach capacity and posture. Human Factors, 50, 211-226.

Chemero, A. (2009). Radical embodied cognitive science. Cambridge, Massachusetts: MIT Press.

Choi, H. J. \& Mark, L. S. (2004). Scaling affordances for human reach actions. Human Movement Science, 23, 785-806.

Doriot, N. \& Wang, X. (2006). Effects of age and gender on maximum voluntary range of motion of the upper body joints. Ergonomics, 49, 269-281.

Gardner, D. L., Mark, L. S., Ward, J. A., \& Edkins, H. (1997). How do task characteristics affect the transition between seated and standing reaches? Ecological Psychology, 13, 245-274.

Gibson, J. J. (1966). The senses considered as perceptual systems. Boston, Massachusetts: Houghton Mifflin. 
Gibson, J. J. (1979). The ecological approach to visual perception. New Jersey: Erlbaum. Jiménez, Á. A. \& Aparicio, C. F. (2009). Sensitivity, changeover responses, and choice in transition. Behavioural Processes, 82, 1-6.

Jiménez, Á. A. \& Aparicio, C. F. (2014). Choice in transition, changeover response requirements, and local preference. The Psychological Record, 64, 31-40.

Kelso, J. A. S (1995). Dynamic patterns: The self-organization of brain and behavior. Cambridge: MIT press.

Kelso, J. A. S. \& Schöner, G. (1988). Self-organization of coordinative movement patterns. Human Movement Science, 7, 27-46.

Konczak, J., Meeuwsen, H. J., \& Cress, M. E. (1992). Changing affordances in stair climbing: The perception of maximum climbability in young and older adults. Journal of Experimental Psychology: Human Perception and Performance, 18, 691-697. Lopresti-Goodman, S. M., Richardson, M. J., Baron, R. M., Carello, C., \& Marsh, K. L. (2009). Task constrains on affordance boundaries. Motor Control, 13, 69-83. Mark, L. S., Nemeth, K., Gardner, D. Dainoff, M. J., Paasche, J., Duffy, M., \& Grandt, K. (1997). Postural dynamics and the preferred critical boundary for visually guided reaching. Journal of Experimental Psychology: Human Perception and Performance, 23, 1365-1379.

Morris, E. K. (2009). Behavior analysis and ecological psychology: Past, present, and future. A review of Harry Heft's ecological psychology in context. Journal of the Experimental Analysis of Behavior, 92, 275-304.

Newell, K. M., McDonald, P. V., \& Baillargeon, R. (1993). Body scale and infant grip configurations. Developmental Psychobiology, 26, 195-205.

Rosas, J. M., Todd, T. P., \& Bouton M. E. (2013). Context change and associative learning. WIREs Cognitive Science, 4, 237-244.

Timberlake, W. (1993). Behavior systems and reinforcement: An integrative approach. Journal of the Experimental Analysis of Behavior, 60, 105-128.

Turvey, M. T., Solomon, H. Y., \& Burton, G. (1989). An ecological analysis of knowing by wielding. Journal of the Experimental Analysis of Behavior, 52, 387-407.

Warren, W. H. (1984). Perceiving affordances: visual guidance of stair climbing. Journal of Experimental Psychology: Human Perception and Performance, 10, 683-703.

Warren, W. H. (1995). Constructing an econiche. En J. Flach, P. Hancock, J. Caird, \& K. Vicente (Eds.), Global perspectives on the ecology of human-machine systems: Vol. 1. Hillsdale, New Jersey: Erlbaum.

Warren, W. H. (2006). The dynamics of perception and action. Psychological Review, 113, 358-389.

Warren, W. H. \& Whang, S. (1987). Visual guidance of walking through apertures: Body scaled information for affordances. Journal of Experimental Psychology: Human Perception and Performance, 13, 371-383. 


\section{Apéndice A}

Resultados individuales de los hombres. El eje de la ordenada representa la proporción de uso de los modos de acción. El eje de la abscisa representa la distancia del bloque expresada en métrica intrínseca.

S1

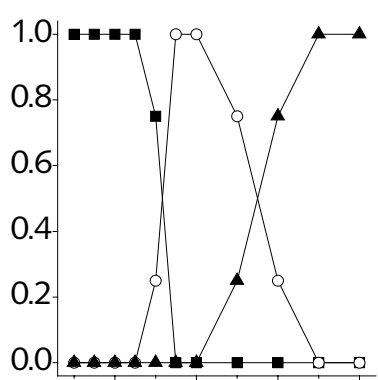

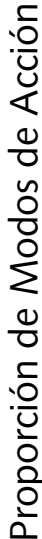

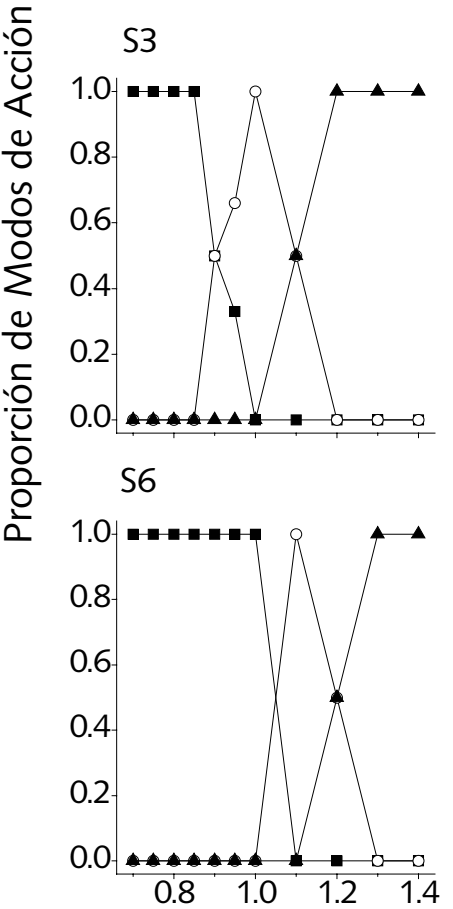

S2

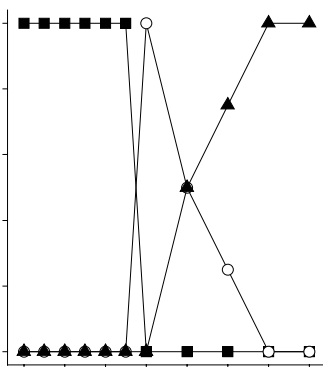

S4

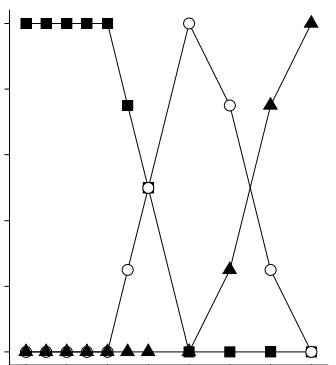

S7

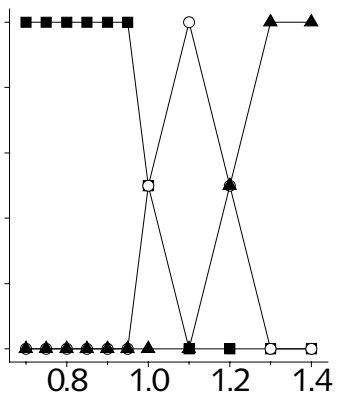

Hombres

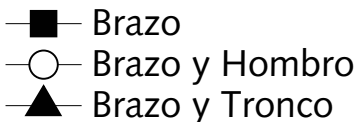

S5

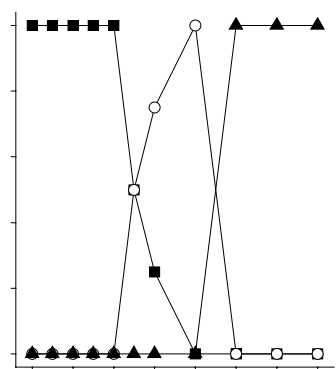

S8

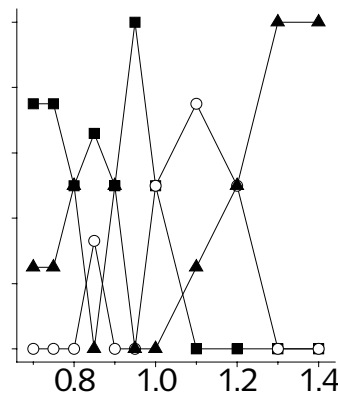

Distancia del Bloque / Longitud del Brazo 


\section{Apéndice B}

Resultados individuales de las mujeres. El eje de la ordenada representa la proporción de uso de los modos de acción. El eje de la abscisa representa la distancia del bloque expresada en métrica intrínseca.

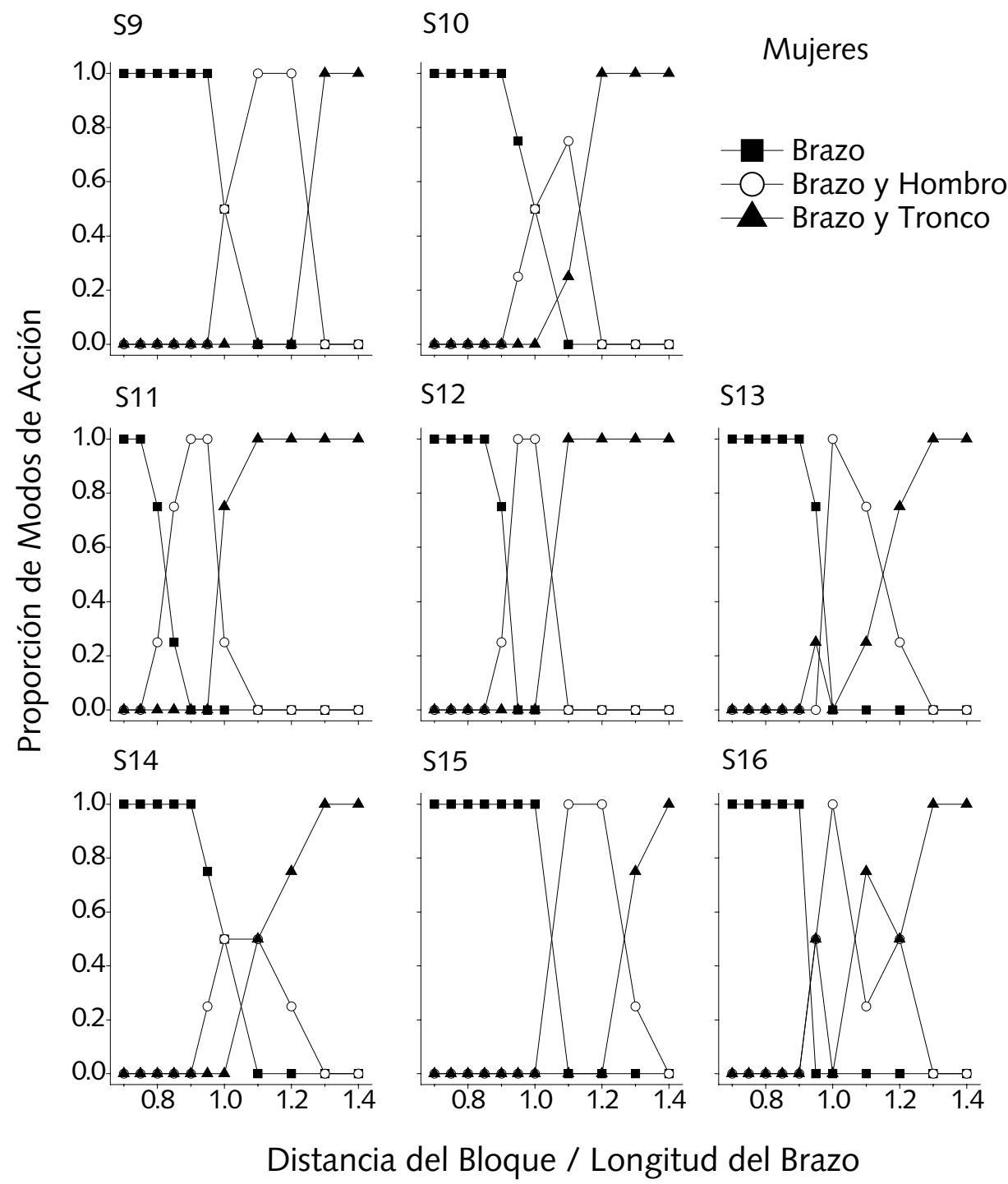

